\title{
Listeria monocytogenes associated with pyogenic spondylitis in a 92-year-old woman
}

\author{
Kento Furuya MD, Naoya Itoh MD DTM\&H
}

Cite as: CMAJ 2021 November 22;193:E1771. doi: 10.1503/cmaj.210205



92-year-old woman with a history of hypertension presented to the hospital with a 1-day history of fever and back pain. One month earlier, she had been admitted to hospital for a L1 compression fracture, and 1 week before the current presentation, she had had nonbloody diarrhea for 3 days. On examination, she was febrile $\left(38.3^{\circ} \mathrm{C}\right)$ and had $\mathrm{L} 1-\mathrm{L} 2$ tenderness but no signs of meningeal inflammation. She had a normal leukocyte count, and mildly raised $\mathrm{C}$-reactive protein and erythrocyte sedimentation rate.

We admitted the patient to the hospital with a differential diagnosis of meningitis, pyogenic spondylitis and epidural abscess. A magnetic resonance imaging (MRI) scan of her vertebra was consistent with pyogenic spondylitis at L1/2 (Figure 1) and cerebrospinal fluid analysis was normal, ruling out meningitis. Listeria monocytogenes was isolated from blood cultures, and we commenced ampicillin on day 2 of her hospital stay. After 2 weeks of antimicrobial therapy, her inflammatory markers improved but her back pain worsened. On day 18 , gadolinium contrast-enhanced MRI showed an epidural abscess at L2. We prescribed 8 weeks of antibiotic therapy (6 wk ampicillin and 2 wk amoxicillin) for L. monocytogenes associated with pyogenic spondylitis and epidural abscess. ${ }^{1}$ Her pain improved from the second week of treatment, and she was able to walk at the time of discharge.

Listeriosis, most often caused by food-borne L. monocytogenes, has been associated with a mortality rate of $30 \% .^{2}$ Patients usually present with fever and diarrhea, but symptoms suggestive of meningitis may occur. Bony infection, as in our patient, is uncommon. ${ }^{2,3}$ In Canada, listeriosis is a notifiable disease, with 158 cases of invasive disease reported in $2018 .{ }^{4}$ Risk factors include advanced age, immunodeficiency and immunocompromise, liver cirrhosis, diabetes and pregnancy. ${ }^{2,5}$ Foods from which $L$. monocytogenes may be acquired include fresh produce, hot dogs, delicatessen meats and unpasteurized milk products; adequate preparation and cooking prevents transmission. ${ }^{5}$ Our patient did not consume dairy products, but her diet was high in raw vegetables, including tomatoes, ginseng, lettuce and cabbage; therefore we were not able to determine the exact source of infection.

\section{References}

1. Berbari EF, Kanj SS, Kowalski TJ, et al. Executive summary: 2015 Infectious Diseases Society of America (IDSA) clinical practice guidelines for the diagnosis and treatment of native vertebral osteomyelitis in adults. Clin Infect Dis 2015;61:859-63.

2. Charlier C, Perrodeau É, Leclercq A, et al. Clinical features and prognostic factors of listeriosis: the MONALISA National Prospective Cohort Study. Lancet Infect Dis 2017;17:510-9.

3. Charlier C, Leclercq A, Cazenave B, et al. Listeria monocytogenes-associated joint and bone infections: a study of 43 consecutive cases. Clin Infect Dis 2012;54:240-8.

4. Reported cases from 1924 to 2018 in Canada: notifiable diseases on-line. Ottawa: Public Health Agency of Canada; modified 2021 Jan. 14. Available: https://dsol-smed.phac-aspc.gc. $\mathrm{ca} /$ notifiable/charts?c=pl (accessed 2021 May 3).

5. Marder EP, Griffin PM, Cieslak PR, et al. Preliminary incidence and trends of infections with pathogens transmitted commonly through food: foodborne diseases active surveillance network, 10 U.S. sites, 2006-2017. MMWR Morb Mortal Wkly Rep 2018;67:324-8.

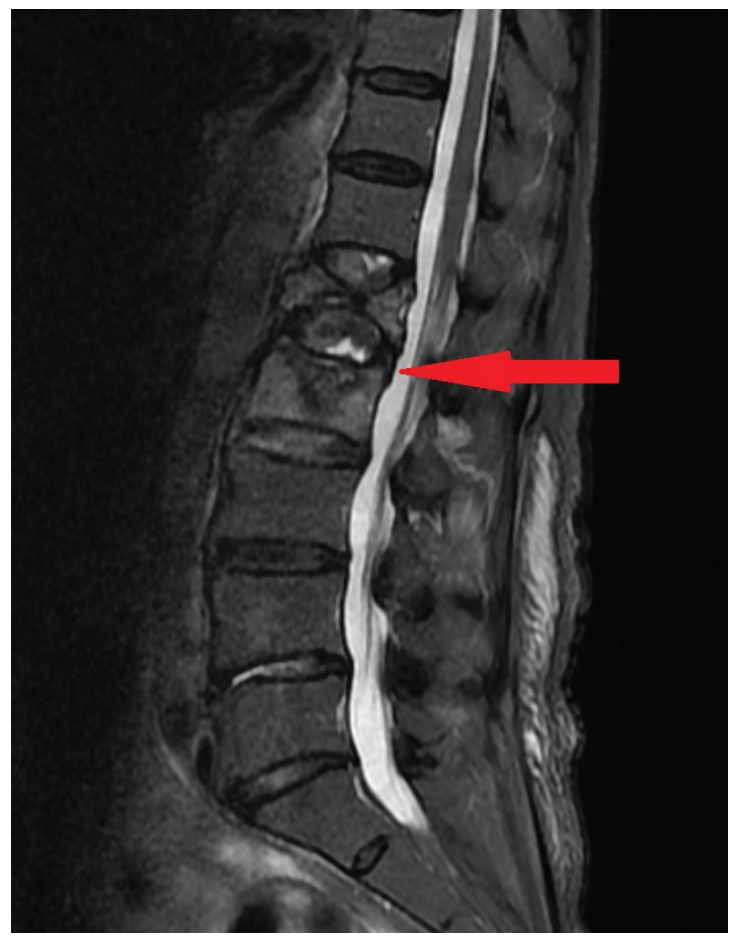

Figure 1: Fat suppressed, weighted magnetic resonance imaging of the lumbar spine of a 92-year-old woman, showing hyperintensity of the $L 1 / 2$ vertebrae and discus, consistent with pyogenic spondylitis.

Competing interests: None declared.

This article has been peer reviewed.

The authors have obtained patient consent.

Affiliations: Department of internal medicine (Furuya), Izu Red Cross Hospital, Kodachino, Izu City, Shizuoka, Japan; Division of Infectious Diseases (Itoh), Aichi Cancer Center Hospital, Chikusa-ku, Nagoya, Japan

Content licence: This is an Open Access article distributed in accordance with the terms of the Creative Commons Attribution (CC BY-NC-ND 4.0) licence, which permits use, distribution and reproduction in any medium, provided that the original publication is properly cited, the use is noncommercial (i.e., research or educational use), and no modifications or adaptations are made. See: https://creativecommons.org/licenses/by-nc-nd/4.0/

Correspondence to: Naoya Itoh, itohnaoya0925@ybb.ne.jp 